\title{
¿Es posible un cambio de actitudes hacia un modelo de desarrollo sostenible?
}

\author{
Caurín Alonso, Carlos \\ Morales Hernández, Antonio José \\ Solaz Portolés, Juan José
}

Departamento de Didáctica de las Ciencias Experimentales y

Sociales de la Universidad de Valencia

Resumen: En la presente investigación se pretende conseguir que unos estudiantes que, en líneas generales, siguen las líneas de un desarrollismo o de un conservacionismo, modifiquen su actitud hacia un modelo más cercano al desarrollo sostenible; para ello se realizan actividades basadas en un taller ambiental y en conocimientos que fomentan el desarrollo sostenible, obteniendo resultados diversos que combinan los conocimientos sobre ciencias sociales y economía con los ambientales. Para realizarlo, seleccionamos estudiantes de distintas etapas de secundaria con características y contextos diferentes. Surge así una idea del desarrollo sostenible múltiple según las situaciones y contextos.

Palabras clave: desarrollo sostenible, educación ambiental, actitudes.

Abstract: In this research is to get some students, in general, follow the lines of a developed or a conservationist, change their attitude towards a model closer to sustainable development; for it is made in a workshop based activities and environmental on knowledge that promote sustainable development, obtaining results that combine various knowledge in social sciences and economy with the environment. To do this, select students from different stages of secondary features and different contexts. Thus emerges a multiple vision of sustainable development according to the situations and contexts.

Key Words: sustainable development, environmental, education, attitude.

(Fecha de recepción: abril, 2012, y de aceptación: septiembre, 2012)

DOI: 10.7203/DCES.26.1926 


\section{Introducción}

En los últimos años, la educación Ambiental se ha convertido en algo muy necesario para comprender, valorar y conservar el Medio Ambiente, considerando que el hombre también es obra y artífice del medio que le rodea.

Una de las finalidades de la Educación Ambiental (EA) es proporcionar a todos el sentido de los valores, las actitudes, el interés activo y las aptitudes necesarias para proteger y mejorar el Medio Ambiente. En Educación Ambiental los contenidos conceptuales pensamos que deberían estar apoyados en conocimientos de la disciplina ecológica, para poder comprender mejor las razones que justifiquen determinadas conductas, pero también son necesarios conocimientos sobre economía, ética, ciencias sociales..., es decir, todos aquellos relacionados con las competencias. Una de las novedades más relevantes del nuevo marco educativo nacional es la introducción de las competencias básicas en el currículo quedando establecido en el artículo 6 de la LOE (2/2006), entendiéndose por currículo, el conjunto de objetivos, competencias básicas, contenidos, métodos pedagógicos y criterios de evaluación de cada una de las enseñanzas. En el Anexo I del Real Decreto 1513/07 define las COMPETENCIAS como aquellos aprendizajes que se consideran imprescindibles, desde un planteamiento integrador y orientado a la aplicación de los saberes adquiridos. Son competencias que toda persona necesita para su desarrollo y satisfacción personal, integración y empleo. Deben estar desarrolladas al finalizar la escolarización obligatoria. Son ocho: Competencia en comunicación lingüística, Competencia matemática, Competencia en el conocimiento y en la interacción con el mundo físico, Tratamiento de la información y competencia digital, Competencia social y ciudadana, Competencia cultural y artística, Competencia para aprender a aprender.

Sin descuidar las otras, nuestro trabajo se basó en la tercera competencia que además entendemos como una evolución lógica del tema transversal llamado Educación Ambiental. Las Competencias básicas incluyen el trabajo conjunto de Conceptos, hechos y principios, procedimientos, valores, normas y actitudes. Una de las formas idóneas de trabajar las competencias básicas es a través de la transversalidad. El trabajo en competencias, como una evolución de la Transversalidad, desde múltiples y diversas instancias, se nos impone como algo necesario para el desarrollo integral de las personas e incluso para la supervivencia dentro de nuestro ecosistema.

Algunas reflexiones que relacionan las competencias y los temas transversales (Gavidia, Aguilar y Carratalá, 2011), nos dicen que ambas se parecen en su carácter transversal, en su intención de aproximar los currículos a la realidad del alumnado, de mostrar la funcionalidad de lo que se aprende, de diseñar las actividades tratando de mostrar los aspectos más axiológicos, de mostrar un compromiso de la escuela con el individuo y la sociedad.

Si logramos compaginar la necesidad de progreso con el respeto al medio, y logramos sentirnos parte del ecosis- 
tema habremos comprendido que no somos los dueños del mismo, sino que formamos parte de él. Si comprendemos las razones por las que se dan algunos problemas, sabremos buscar soluciones con sentido. Ese sentido común, basado en una actitud crítica y de respeto es lo que dará a las competencias básicas, como antes a los temas transversales una dimensión de espada e infusión. Reinterpretando a Pujol RM, citado por Caurín (pág 7, 2001), podemos hablar de las competencias como una espada que atraviesa todas las áreas curriculares y hablar de las mismas como una infusión que se distribuye por un líquido (el currículum), de manera, que se forma una disolución homogénea y no se bebe el líquido que no contiene parte de las sustancias disueltas. Todas las partes del currículum trabajan impregnadas con las competencias. Todas deben fomentar el cambio de actitudes hacia las valoraciones críticas y de respeto por el medio y la sociedad.

Por ello y teniendo como ejemplos de modelos a seguir estas perspectivas intentamos medir la evolución de actitudes en unos estudiantes hacia modelos de consenso tras seguir diversos tratamientos.

Por otro lado, al realizar un programa de Educación Ambiental, pretendemos fomentar actitudes hacia el medio ambiente que se encuentre dentro del modelo de desarrollo sostenible. El término Desarrollo Sostenible está en todos los programas y currícula de Educación Ambiental. La idea clásica de Desarrollo Sostenible intenta abordar la relación entre desarrollo y medio ambiente. En 1972 (Caurín 2007) se constituye un grupo internacional de distinguidos empresarios, estadistas y científicos que se bautizó como Club Roma. Escandalizaron al mundo cuando escribieron un libro titulado, "Los límites del crecimiento". Allí se planteaban las siguientes cuestiones: ¿Qué pasaría si el crecimiento de la población mundial siguiera sin control? ¿Cuáles serían las consecuencias medioambientales si el desarrollo económico continuara a su paso actual? ¿Qué se puede hacer para asegurar una economía humana que provea lo suficiente para todos y que además tenga cabida dentro de los límites físicos de nuestro planeta? Sus conclusiones fueron catastrofistas, pero veinte años más tarde, el Club de Roma vuelve a editar un libro "Más allá de los límites de Crecimiento" donde actualizarán sus propuestas, dando un tratamiento enérgico para la curación del mundo y defendiendo el modelo de Desarrollo Sostenible.

En 1983, la Asamblea General de la Naciones Unidas creó la Comisión Mundial sobre Medio Ambiente y Desarrollo, presidido por Gro Harlem Brundtland, en el que, frente al concepto de crecimiento cero inicial del Club de Roma, propone el de Desarrollo Sostenible

En la Conferencia o Cumbre de Río (1992) en su principio 21 apela a la “... creatividad, los ideales y el valor de los jóvenes... (para)... lograr un desarrollo sostenible...” (Declaración de Río, 1992, en Almenar et al., 2000:28)

El concepto desarrollo sostenible tiene también grados y hablamos de modelos en general porque cuestionamos que exista un único modelo de Desarrollo Sostenible (Caurín, 1999). 
Respecto al cambio de actitudes, podemos decir que Según Rokeach (1973), existen mayores probabilidades de conseguir un cambio de actitudes, valores y conducta en el individuo si su concepto de sí mismo se ve afectado por la nueva información presentada, y experimenta una sensación de desencanto consigo mismo. Esta sensación puede producir un cambio de valores duradero (Rokeach, 1973).

Otros trabajos hablan se refieren al fomento de la actitud crítica ante problemas y situaciones ambientales, tiene referentes en el planteamiento de situaciones ambientales ante las que hay que tomar decisiones: dilemas morales, comprensión crítica de la realidad (Caurín, Gil 1995, Caurín 1999, Hernández, Caurín y Llopis 2005). También la llamada inculcación pretende que el alumno adopte unos valores determinados, o que modifique valores ya existentes (Superka, 1976, Santoyo, 1999). El reforzamiento positivo y negativo se basa en el premio o castigo de las conductas. Mediante la dramatización los alumnos deben adoptar y defender distinto puntos de vista. A partir del modelo de profesor investigador (Xouto, 2010) es posible, además, analizarlo de forma científica.

\section{Objetivos e hipótesis}

Los objetivos de investigación son:

- Descubrir las actitudes previas a la aplicación de un programa de Educación Ambiental (EA) y ver en qué medida han cambiado tras la misma.
- Modificar o fomentar las actitudes de los estudiantes hacia un modelo de desarrollo sostenible.

La hipótesis está basada en la idea de que para modificar actitudes es necesario conocer el objeto a que se refiere la actitud. No se pueden modificar las actitudes hacia un objeto o aspecto determinado si antes no conocemos ampliamente sus características. En nuestra investigación, el objeto de la actitud es el medio ambiente y el procedimiento para conocerlo la educación ambiental. Debemos integrar contenidos ambiéntales con aspectos socioeconómicos y morales. Teniendo en cuenta esto, nuestra hipótesis sería la siguiente:

"Para producir un cambio de actitudes hacia un modelo de Desarrollo Sostenible es condición necesaria la adquisición de un conocimiento profundo de los fenómenos ecológicos y ambientales, integrados con aspectos socioeconómicos y morales sobre los que fundamentar criterios razonables.”

\section{Metodología}

Para realizar nuestra investigación consideramos necesario hallar la forma de desarrollar la competencia en el conocimiento y en la interacción con el mundo físico. Para ello, nos basamos en un Proyecto de Educación Ambiental que obtuvo un premio Educación y Sociedad del Ministerio de Educación (Caurín, Sigler ). Este proyecto se realizó con actividades en torno a tres grandes temas que elegimos: Contaminación, Reciclado y Energía y un cuarto apartado que englobaba los tres aspec- 
tos de manera interdisciplinar. Escogimos estos temas por tres motivos: a) Su cercanía a la vida diaria; b) La relación existente entre los tres aspectos; c) La interdisciplinariedad que permite entre distintos departamentos y su gran enfoque y relación en los temas de ciencia tecnología y sociedad.

Se realizaron todas las actividades, pero para hacer una valoración del estado inicial y del estado final, decidimos centrarnos en el cuarto apartado, por englobar actividades que hacen referencia a los tres aspectos. Para ello se decidió confeccionar unos cuestionarios previos y posteriores a la aplicación del proyecto mencionado anteriormente $\mathrm{y}$ elegir una muestra de alumnos adecuada a nuestra investigación.

\section{La muestra seleccionada fue la siguiente:}

Grupo 1: Se realizó en un instituto de una localidad rural, con alumnado de $2^{\circ}$ de bachillerato que habían escogido la asignatura de Ciencias de la Tierra y del Medio Ambiente, materia que contempla la Ecología como ciencia base y que, además permitía incluir en la programación el proyecto de Educación Ambiental al completo. Los alumnos de este curso de bachillerato apenas habían recibido conceptos de ecología debido a la organización de los departamentos del centro que habían priorizado otro tipo de contenidos. El grupo objeto de estudio constó de 20 estudiantes con edades comprendidas entre los 16 y 19 años.

Grupo 2: eran alumnos que no habían recibido conocimientos conceptuales de ecología e iban a recibir algunos temas de ecología a la vez que se aplicaba el programa de Educación Ambiental de forma transversal. La experiencia se realizó en un instituto de zona industrial en un curso de $3^{\circ}$ de ESO. Pretendíamos que a la vez que realizábamos el programa, se impartieran conocimientos someros de ecología. Se incluyó el programa de Educación Ambiental, como apoyo a la programación de Ciencias Naturales. Este grupo constó de 20 estudiantes con Es un curso de edades comprendidas entre los 14 y 17 años; tampoco habían trabajado temas ambientales.

Grupo 3: eran alumnos que no habían recibido conocimientos conceptuales de ecología y no iban a recibirlos durante la época en que se aplicaba el programa de Educación Ambiental. La experiencia se realizó en un centro de secundaria de una localidad rural, agrícola e industrial y que tiene una central térmica en las proximidades. La investigación tenía como base un taller de Educación Ambiental que se constituyó como actividad extraescolar voluntaria durante dos tardes a la semana (6 horas semanales). Resaltamos el carácter voluntario de los asistentes. Existían en el grupo que realizaba el taller varios niveles educativos mezclados entre 14 y 18 años. Ninguno de ellos había trabajado conocimientos de ecología.

\section{Métodos de recogida de datos y aná- lisis de los mismos}

En este trabajo se utilizaron como métodos de recogida de datos preguntas abiertas y semiabierts en un dise- 
ño pretest-postest. Eran preguntas que implicaban al alumnado en las respuestas. Para organizar y exponer los datos, se utilizaron las redes sistémicas y las tablas de importancia que permiten estructurar la información para comprenderla y actuar posteriormente sobre ella.

Las redes sistémicas permiten una primera interpretación descriptiva de los datos. Provienen de la lingüística sistemática que empezó sobretodo con Halliday (Beny 1975, 1977, Halliday 1973, 1975, 1978; Kress 1976) citados por Neus Sanmartí (1989) y se ha utilizado en estudios sociológicos (Turner y M. Killian, 1993) y en la comprensión automatizada del lenguaje (Winogrod 1972). También son utilizados en didáctica de las Ciencias y de las Matemáticas por Blis y otros (1979), Sanmartí (1989), Caurín y Gil (1995) y Caurín (1999), Hernández, Caurín y Llopis $(2003,2005)$. Se construye una red para clasificar las respuestas a los cuestionarios. Los alumnos a menudo utilizan distintas palabras para expresar las mismas ideas o las mismas palabras para expresar distintas ideas. Las frases o palabras de los alumnos corresponden a diferentes ítems de la Red sistémica, en la que hemos recogido los diferentes aspectos de las frases. Una vez realizadas las redes sistémicas, se procede a confeccionar las tablas de importancia, Caurín y Gil (1995), Caurín (1999), Caurín, y Sanjosé (2002), Hernández, Caurín y Llopis (2003, 2005), Martínez Penella, Caurín y Sanjosé, (2010), categorizando numéricamentre los distintos aspectos aparecidos en las Redes Sistémicas según la importancia o cohe- rencia de la respuesta. Se confeccionan tablas de categorías a las que daremos un valor basado en un sistema trinario (1, 2 y 3 , siendo el uno el valor menos importante ).

Después se realizó un tratamiento estadístico que describiremos en los resultados.

Para realizar la prueba previa nos basamos en el visionado de un video sobre impacto ambiental y un cuestionario basado en el video. La práctica consiste en la observación de un vídeo sobre Australia en el que se tratan los siguientes impactos: Tala incontrolada de árboles, incendios forestales, salinización y desertización del suelo, plagas, sequía...

El cuestionario siguiente, basado en la actividad, lo utilizamos como pre-test en nuestro trabajo:

Como estudiante de inglés en Espa$\tilde{n} a$, recibes en tu casa a un estudiante australiano, con inquietudes ecologistas, Joseph Winston. Joseph te habla de las plagas que tú has observado en el vídeo; tú le cuentas que en España esas cosas ocurren en otros niveles, aunque otras se dan exactamente igual. Reflexiona $y$ responde a estas preguntas:

1) De los problemas que te ha nombrado Joseph, ¿cuáles se dan en tu comarca?. ¿Y en tu país?. ¿Cuáles se dan en tu país y comarca y no están contemplados en el vídeo?.

2) Joseph te pregunta qué soluciones se aportan en España a los problemas planteados.

3) Siguiendo con su curiosidad, Winston pregunta quién o quiénes son los responsables en tu país o región de solucionar estos 
problemas.¿Quién o quiénes, a tu juicio, deberían hacerlo?.

Para realizar la prueba posterior realizamos una actividad basada en una historia tipo juego de rol (Caurín 2000) en el que los actores han de asumir una serie de personajes. La historia cuenta un supuesto de instalación de una fábrica de papel en su localidad. Los actores se sitúan ante el público tras elegir libremente sus papeles y representan la escena, actuando con la mayor naturalidad y expresándose con su propio lenguaje. El cuestionario y los resultados de la actividad (debate) siguiente, basado en la actividad, lo utilizamos como posttest en nuestro trabajo. El grupo, con la ayuda del profesor analiza los distintos elementos de la situación: ideas, sentimientos, actitudes, soluciones apuntadas. La pregunta final fue: ¿Qué piensas que debe recomendar el Dr. López: 1) Aceptar la fábrica sin reservas, 2) Aceptar la fábrica con reservas (tomando medidas que aseguren una actividad no contaminante), 3) Rechazar la fábrica sin reservas, 4)Rechazar la fábrica con reservas (a menos que quede plenamente garantizada una actividad no contaminante en absoluto).

Antes de tomar tu propia decisión piensa en los posibles hechos o alternativas que pueden incidir en ella.

\section{Resultados}

Tras la actividad previa, confeccionamos las redes sistémicas y las tablas de importancia

Descripción e interpretación de la Red Sistémica del grupo 1 (figura 1)
Resaltamos algunos puntos:

Gran parte de los alumnos (65\%) piensan que las causas de los impactos ambientales son debidas a acciones humanas más que a causas naturales. La mayor parte de las soluciones propuestas so de tipo ambientalista (95\%). Las medidas de ahorro referidas al agua o la energía tienen poco peso (alrededor de un 30\% ). Hay también bastantes soluciones atribuibles a concepciones de Desarrollo sostenible, aunque la mayor parte de ellas se refiere a la reparación del medio Tal vez está en la conciencia de los ciudadanos el hecho de que reparando el daño se solucionan muchas problemas; esto puede evitar desarrollar acciones de prevención; además se infiere de muchas respuestas una confusión entre reaprovechamiento y reciclado. El 75\% de los encuestados piensan que son responsables de los problemas del Medio Ambiente, aunque sólo un 35\% proponen acciones concretas. Respecto al $25 \%$ que traslada esa responsabilidad, destaca el $15 \%$ que echa la culpa al gobierno (ver figura 1).

Descripción e interpretación de la Red Sistémica del grupo 2

- En este grupo, apenas se nombran causas de los impactos (35\%), aunque siguen apareciendo sobre todo las causas debidas a la acción humana. Los tipos de soluciones no presentan grandes diferencias entre ellos (ambientales, económicas), aunque predominan las ambientales (55\%). En las de desarrollo sostenible predominan ligeramente las de prevención frente a las de reparación. 
Figura 1.

\section{Red sistémica de las respuestas correspondientes al grupo 1}

Network Impacto ambiental en Australa Grupo 1

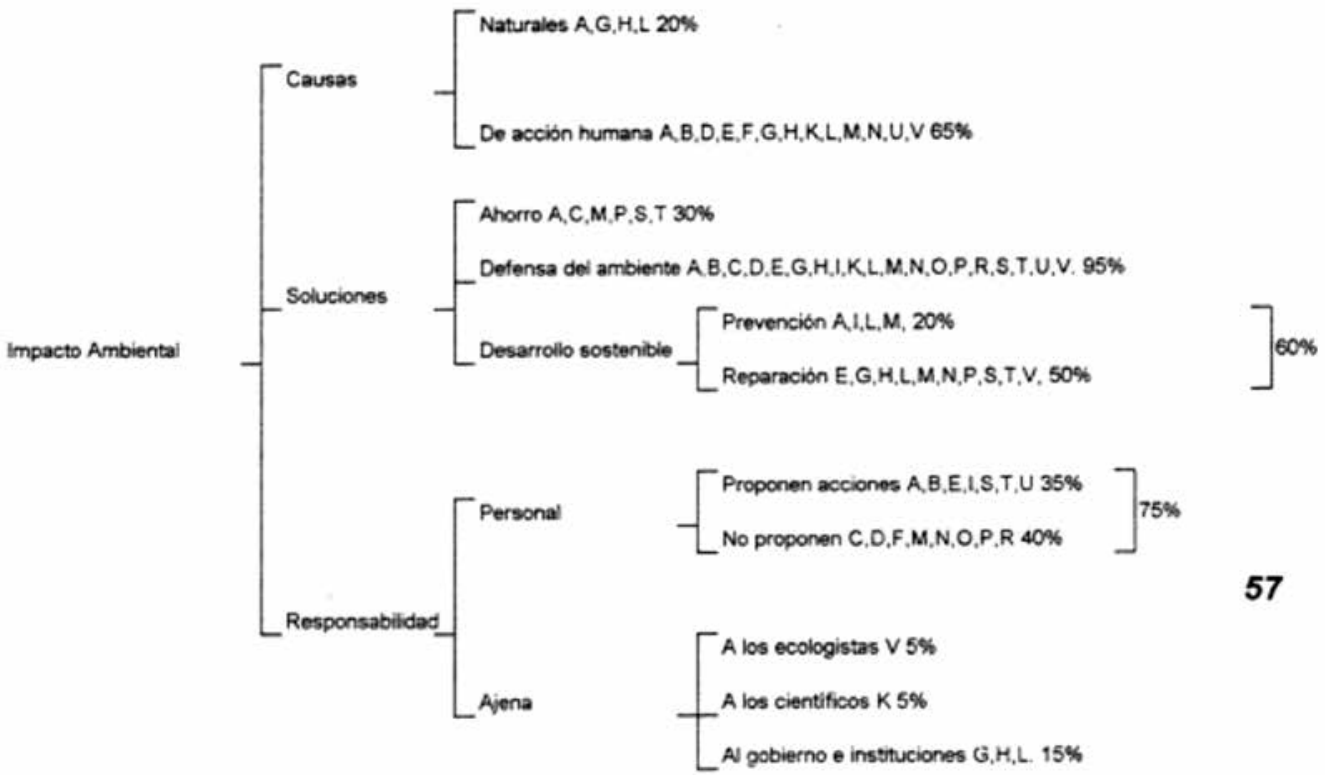

Fuente: Elaboración propia

- El 55\% de los encuestados no se implican en las responsabilidades frente al Medio Ambiente, pero sí lo hace el $45 \%$, aunque sin proponer acciones concretas en su mayor parte. Si comparamos este grupo con el grupo 1, observamos algunas diferencias:

- Los alumnos del grupo 1 describen muchas más causas que los alumnos del grupo 2

- El rango de soluciones es más amplio en el grupo 1 ( $95 \%$ frente al $55 \%$ del grupo 2)

- También la implicación personal es mayor en el grupo 1.
- El grupo 2 hace recaer en mucha mayor medida la responsabilidad sobre el gobierno e instituciones.

Descripción e interpretación de la Red Sistémica del grupo 3

De la red sistémica construida, destacamos que estos sujetos:

- Consideran que las causas de los impactos son debidas principalmente a la acción humana.

- Predominan la falta de soluciones, pero los que proponen alguna, se refieren a soluciones de tipo ambiental. 
- Predominan más las respuestas conservacionistas que las referidas a un desarrollo incontrolado.

- Este cuestionario da unas respuestas mucho menos definidas y más pobres en conceptos que el cuestionario de los grupos 1 y 2 .

\section{Descripción estadística de los datos}

Para poder describir de forma cuantitativa los resultados, confeccionamos las tablas de importancia que miden aspectos similares en el pre-test y en el post-test. Estas tablas están basadas en categoría de importancia y han sido construidas, en el caso del pre test haciendo una nueva lectura de los datos y complementando éstos con las redes sistémicas.

Para el post-test, al ser un cuestionario semicerrado ha sido más fácil construir la tabla basándose en el orden de importancia que se daba a cuestiones relacionadas con los aspectos ambientales o económicos que estamos analizando.

PRE-TEST: Las tablas han sido construidas según tres órdenes de importancia:
- No dan ninguna importancia al aspecto en cuestión. Lo ignoran o menosprecian

- Dan una cierta importancia al aspecto en cuestión, pero sin énfasis o apoyo explícito. Se trata de situaciones que podríamos definir como neutras, sin juicios de valor explícitos.

- Dan mucha importancia al aspecto en cuestión, mencionando o subrayando la relevancia.

Los aspectos contemplados son:

Deforestación, desertización, reciclado, Consumo de agua y sequía, Contaminación, Repoblación inadecuada (incendios), Energía y ahorro energético, Economía.

\section{POST-TEST}

Este cuestionario valora la importancia que se le da a algunas frases informativas referidas a aspectos ambientales y económicos (valorando aquellos aspectos que hemos valorado en el pre-test). Pondremos como ejemplo las tablas de importancia del grupo 1 (Figuras 2 y 3 )

\section{Figura 2.}

TEST SOBRE EL VIDEO DE IMPACTO AMBIENTAL EN AUSTRALIA
\begin{tabular}{|c|c|c|c|c|c|c|c|c|c|c|c|c|c|c|c|c|c|c|c|c|}
\hline & (GRUPO 1 ) \\
\hline $\begin{array}{c}\text { DEFORESTACION } \\
\text { DESERTIZACION RECICLAD }\end{array}$ & 3 & 3 & 2 & 3 & 3 & 1 & 3 & 2 & 2 & 3 & 3 & 3 & 3 & 1 & 3 & 1 & 2 & 3 & 1 & 3 \\
\hline $\begin{array}{c}\text { CONSUMO DE AGUAY } \\
\text { SEQUIA }\end{array}$ & 3 & 3 & 1 & 1 & 3 & 1 & 3 & 3 & 1 & 3 & 3 & 3 & 1 & 3 & 3 & 1 & 2 & 2 & 3 & 1 \\
\hline CONTAMINACION & 3 & 2 & 1 & 3 & 1 & 3 & 2 & 3 & 3 & 2 & 3 & 3 & 3 & 1 & 1 & 3 & 1 & 3 & 3 & 3 \\
\hline $\begin{array}{c}\text { REPOBLACION INADECUADA } \\
\text { (NCENDIOS) }\end{array}$ & 3 & 3 & 3 & 3 & 3 & 3 & 3 & 3 & 3 & 3 & 3 & 1 & 3 & 3 & 3 & 3 & 3 & 3 & 3 & 3 \\
\hline $\begin{array}{c}\text { ENERGIAYAHORRO } \\
\text { ENERGETICO }\end{array}$ & 3 & 3 & 1 & 1 & 1 & 3 & 1 & 1 & 3 & 1 & 1 & 1 & 1 & 1 & 1 & 1 & 1 & 1 & 3 & 1 \\
\hline ECONOMIA & 3 & 1 & 1 & 2 & 1 & 1 & 1 & 1 & 1 & 1 & 1 & 1 & 3 & 1 & 1 & 1 & 2 & 1 & 1 & 2 \\
\hline
\end{tabular}

Fuente: Elaboración propia 


\section{Figura 3.}

TEST SOBRE LA INSTALACIÓN DE UNA FÁBRICA PAPELERA

\begin{tabular}{|c|c|c|c|c|c|c|c|c|c|c|c|c|c|c|c|c|c|c|c|c|}
\hline & $\bar{A}$ & 8 & C & $\mathrm{D}$ & $E$ & $F$ & $G$ & $\mathrm{H}$ & 1 & $\mathrm{~K}$ & $\mathrm{~L}$ & $\mathbf{M}$ & $\mathbf{N}$ & 0 & $\bar{P}$ & $R$ & S & $\mathrm{T}$ & $\bar{U}$ & $\bar{V}$ \\
\hline $\begin{array}{c}\text { DEFORESTACION } \\
\text { DESERTIZACION RECICLADO }\end{array}$ & 3 & 3 & 3 & 3 & 3 & 3 & 2 & 3 & 3 & 2 & 3 & 3 & 3 & 3 & 3 & 3 & 3 & 3 & 3 & 3 \\
\hline $\begin{array}{l}\text { CONSUMO DE AGUAY } \\
\text { SEQUIA }\end{array}$ & 3 & 3 & 2 & 3 & 3 & 3 & 2 & 2 & 3 & 3 & 3 & 3 & 2 & 3 & 3 & 3 & 3 & 3 & 3 & 3 \\
\hline CONTAMINACION & 3 & 3 & 3 & 3 & 3 & 3 & 3 & 3 & 3 & 3 & 3 & 3 & 3 & 3 & 3 & 3 & 3 & 3 & 3 & 3 \\
\hline $\begin{array}{l}\text { REPOBLACION INADECUADA } \\
\text { (INCENDIOS) }\end{array}$ & 3 & 3 & 2 & 3 & 3 & 3 & 3 & 3 & 3 & 2 & 3 & 3 & 3 & 3 & 3 & 3 & 3 & 3 & 3 & 3 \\
\hline $\begin{array}{c}\text { ENERGLAY YHORRO } \\
\text { ENERGETICO }\end{array}$ & 2 & 3 & 2 & 3 & 3 & 1 & 2 & 2 & 1 & 3 & 3 & 3 & 3 & 3 & 3 & 3 & 3 & 3 & 3 & 3 \\
\hline \multicolumn{21}{|l|}{ ECONOMIA } \\
\hline COSTE ECONOMICO & 3 & 2 & 3 & 2 & 3 & 2 & 1 & 2 & 2 & 2 & 2 & 3 & 1 & 2 & 2 & 3 & 3 & 3 & 3 & 1 \\
\hline EMPLEO & 2 & 3 & 3 & 3 & 3 & 3 & 1 & 3 & 3 & 3 & 3 & 3 & 3 & 3 & 2 & 3 & 3 & 3 & 3 & 3 \\
\hline EXPORTACION & 3 & 1 & 2 & 3 & 3 & 1 & 2 & 1 & 3 & 1 & 2 & 2 & 2 & 2 & 3 & 3 & 3 & 3 & 3 & 3 \\
\hline ESCOGE & $\bar{A}$ & $\bar{B}$ & $\mathrm{C}$ & $\bar{D}$ & $\mathrm{E}$ & $F$ & $G$ & $\mathrm{H}$ & $T$ & $\bar{K}$ & $\mathrm{~L}$ & $\mathrm{M}$ & $\bar{N}$ & 0 & $\bar{P}$ & $\bar{R}$ & $\mathrm{~s}$ & $\mathrm{~T}$ & $\bar{U}$ & $\overline{\mathbf{V}}$ \\
\hline \multicolumn{21}{|l|}{ ACEPTAR SIN RESERVAS } \\
\hline ACEPTAR CON RESERVAS & $\mathrm{X}$ & & $\mathrm{X}$ & & $\mathrm{X}$ & & & $\bar{x}$ & $\bar{x}$ & $\bar{x}$ & $x$ & $\mathrm{x}$ & & $\bar{x}$ & $\mathrm{x}$ & $\mathrm{x}$ & $\bar{x}$ & $\bar{x}$ & $x$ & $\bar{x}$ \\
\hline RECHAZAR SIN RESERVAS & & $\bar{x}$ & & $\bar{x}$ & & $\bar{x}$ & $\bar{x}$ & & & & & & $\mathrm{X}$ & & & & & & & \\
\hline RECHAZAR CONRESERVAS & & & & & & & & & & & & & & & & & & & & \\
\hline
\end{tabular}

Fuente: Elaboración propia

\section{Interpretación gráfica}

Evolución de la variable ambiental (categoría 3) (figura4).

La categoría 3 es la que corresponde a aquellos individuos que le dan mucha importancia al medio ambiente; si los tratamientos son correctos debe aumentar en la gráfica el porcentaje de sujetos con esa valoración.

- GRUPO 1: Observamos que el aumento es muy grande. Desde un $20 \%$ de sujetos pasamos a $90 \%$. Los tratamientos en este caso se confirman como correctos.

- GRUPO 2: Observamos que pasa de $0 \%$ a $15 \%$. Ha mejorado un poco; como confirmación de las tablas , no podemos considerarlo correcto

- GRUPO 3: Observamos también una leve mejoría. Tampoco podemos considerar correctos los tratamientos.
Evolución de la variable económica (categoría 3) (figura 4)

La categoría 3 es la que corresponde a aquellos sujetos que le dan mucha importancia a la economía; si los tratamientos son adecuados, debe aumentar en la gráfica el porcentaje de sujetos con esta valoración.

- GRUPO 1: Vemos que el aumento va de 10 al $50 \%$ tras los tratamientos, lo que podemos considerar correctos

- GRUPO 2: Observamos que hay una mejoría de 0 a $20 \%$ tras los tratamientos; lo podemos considerar un correcto aunque hay un leve mejoría.

- GRUPO 3: Hay un empeoramiento, ya que desaparece la categoría 3 después del tratamiento, que para este grupo han sido inadecuado o incompleto. 


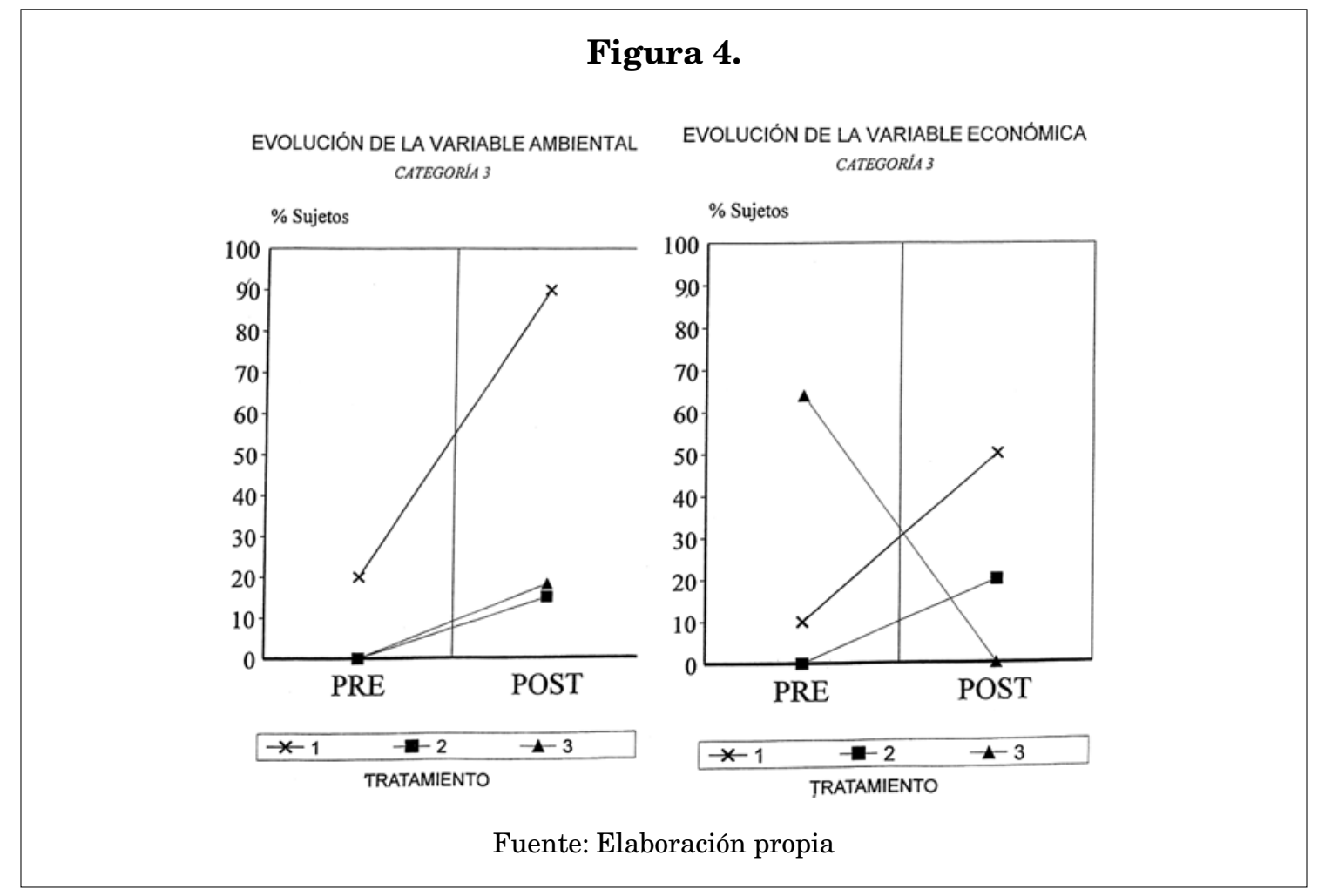

Interpretación de las tablas de variable ambiental y económica combinadas: tablas de Desarrollo Sostenible (figura 5)

Vamos a describir una gráfica clasificatoria y luego otra que nos permite una visión de la evolución de los tres grupos: en estas tablas se hace una aproximación al modelo de desarrollo sostenible que intenta compaginar economía y medio ambiente. Aquí se comparan las coordenadas directas (ambientales/ económicas) para cada grupo. Para ello vamos a definir los diferentes tipos de sujetos que pueden surgir combinando las variables ambiental y económica en pares ordenados. (categoría económica, categoría ambiental).
- Sujetos $(1,1)$ : son alumnos desinteresados, tanto por los problemas ambientales como por los problemas económicos. Tendría un desarrollo sostenible negativo.

- Sujetos $(1,2)$ : son sujetos a los que les importa un poco más el medio ambiente que el aspecto económico, el cual no le importa nada. Tendría también un desarrollo sostenible negativo.

- Sujetos (1,3): son los llamados ecologistas viscerales. Sólo les importa el medio ambiente, y no admiten el desarrollo si este perjudica al medio. Coincide con nuestra descripción de conservacionista acérrimo. 
- Sujetos $(2,1)$ : son sujetos a los que les importa un poco más el aspecto económico que el aspecto ambiental, el cual no les importa nada. Tienen un desarrollo sostenible negativo.

- (2,2): son sujetos esencialmente neutros en todo, es decir, dan una cierta importancia al aspecto, pero sin matizar.

- $(2,3)$ : son sujetos que poseen desarrollo sostenible positivo, pero que tienen un sesgo hacia el aspecto ambiental, al que consideran más importante.

- $(3,1)$ : Son sujetos desarrollistas, que sostienen el modelo de desarrollismo incontrolado, sin importarles nada el medio ambiente.

- $(3,2)$ : Son sujetos que poseen desarrollo sostenible positivo, pero tienen un sesgo hacia el aspecto económico a desarrollista, al que consideran más importante.

- (3,3): Son los que siguen el modelo de desarrollo sostenible positivo ideal, los que consideran muy importante ambos aspectos.

Para entender mejor los diferentes tipos de sujetos con desarrollo sostenible que pueden existir hemos construido una gráfica con dos ejes, un eje de desarrollo sostenible que va de izquierda a derecha y cruzando un eje de sesgo desarrollista conservacionista.

Se pueden apreciar los dominios de los dos sesgos y de los dos desarrollos sostenibles.

El desarrollo sostenible positivo es aquel hacia el cual tiende el tratamiento y corresponde a los sujetos $(3,3)$ el ideal $(3,2)$ con sesgo desarrollista y $(2,3)$ con sesgo conservacionista, aunque los tres tipos están dentro de unos cánones lógicos de desarrollo sostenible.

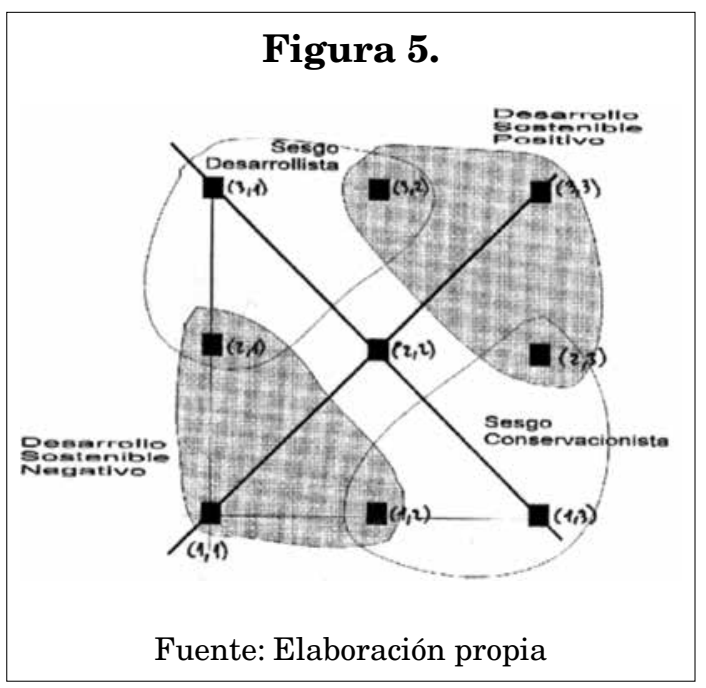

Con ayuda de las gráficas combinadas de las variables ambiental y económica (gráfica de Desarrollo Sostenible positivo y gráfica de cambios en el Desarrollo Sostenible) (figura 7) en el pre y post tratamiento. vamos analizar que grupos han evolucionado en los dos aspectos.

- GRUPO 1: Observamos que en el estado previo se dan los sujetos $(1,2) 60 \%,(1,3) 15 \%,(2,2) 15 \%,(3,2)$ $5 \%$ y $(3,3) 5 \%$. En esencia hay un $10 \%$ de sujetos con desarrollo sostenible positivo en el estado previo. En el estado posterior $45 \%$ pasa a $(2,3), 5 \%$ quedan en $(3,2)$ y $45 \%$ quedan en $(3,3)$. En total un $95 \%$ de sujetos pasan a tener desarrollo sostenible positivo. El éxito del tratamiento ha sido muy notable.

- GRUPO 2: En el estado inicial hay sujetos $(1,1)$ es decir desinteresados 
y un 55\% de $(1,1)$ con desarrollo sostenible negativo. Tras el tratamiento, quedan desarrollo sostenible positivo las siguientes $10 \%$ en $(2,2)$, $15 \%$ en $(3,2)$ y $5 \%$ en $(3,3)$. En total evolucionan desde 0 a $30 \%$ de desarrollo sostenible positivo. El tratamiento no ha sido un éxito, pero ha mejorado bastante el modelo de desarrollo sostenible del grupo.
- GRUPO 3: En el estado inicial observamos un $27,3 \%$ de sujetos e el estado de desarrollo sostenible positivo, aunque con sesgo economicista. Tras el tratamiento, se reducen el número de sujetos con desarrollo sostenible positivo a $18,2 \%$ manteniendo el mismo sesgo. El tratamiento aplicado a este grupo no ha sido el adecuado.
EVOLUCIÓN DE DESARROLLO SOSTENIBLE POSITIVO

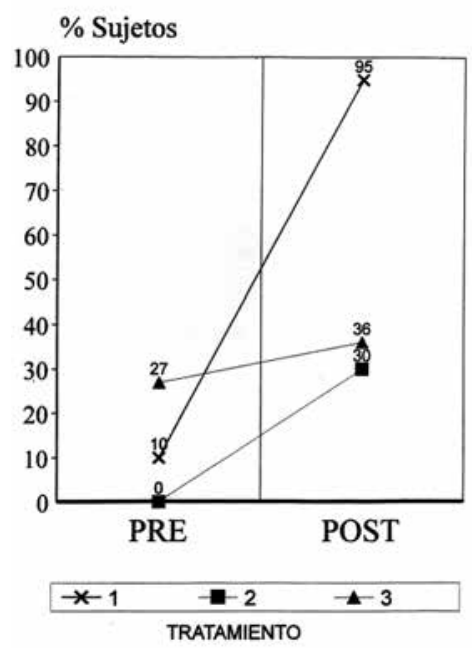

\section{Figura 6.}

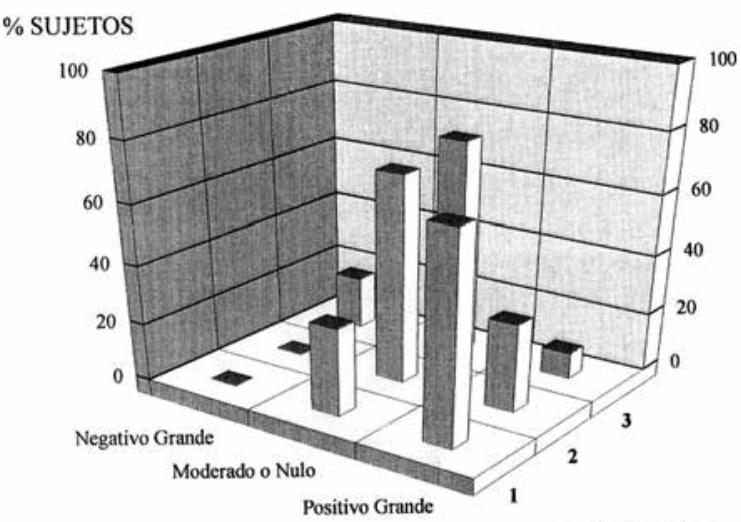

CAMB. DES.SOSTENIBLE
TRATAMIENTO

Fuente: Elaboración propia

\section{Prueba de la mediana}

Vamos a analizar los datos con un tratamiento estadístico basado en el test de la mediana. Se llama mediana de una distribución estadística a aquel valor que ocupa el lugar central al situar ordenadamente todos los datos o valores de la distribución.

En la tabla "Cambios en desarrollo sostenible" (figura 8) se aplica el test de la mediana a los cambios en desarrollo sostenible. La mediana de los cambios es 1 , y podemos observar :

- En el grupo 1 hay 14 sujetos que han cambiado por encima de la mediana.

- En el grupo 2 sólo hay 6 sujetos que han cambiado por encima de la mediana.

- En el grupo 3 sólo hay un sujeto que ha cambiado por encima de la mediana. 


\section{Figura 7. Cambios en Desarrollo Sostenible (por grupo)}

\section{TRATAMIENTO}

\begin{tabular}{|l|l|l|l|}
\hline Grupos & 1 & 2 & 3 \\
\hline GT Median & 14 & 6 & 1 \\
\hline LE Median & 6 & 14 & 10 \\
\hline
\end{tabular}

Casos: 51 Mediana: 1 Chi-Square: 12,5668 DF: 2 Significación: 0,0019

Fuente: Elaboración propia

Se confirma que el mayor número de sujetos que ha cambiado ha sido el del grupo 1.

El test de la mediana y el estadístico $\mathrm{X}^{2}$ no nos demuestran estadísticamente que ha sido correcto el tratamiento, sino que los resultados se deben al tratamiento y no al azar.

\section{Discusión}

Del análisis de las redes sistémicas aplicados en la categorización de las respuestas de los cuestionarios previos, podemos deducir algunas ideas:

Los alumnos del grupo 1, como ya hemos comentado al describir el Network, expresan más medidas de reparación que de prevención. El hecho de reciclar el vidrio o el papel, puede haber creado la equivocada idea de que es no hay ningún problema en consumir todo el papel o vidrio que queramos porque luego se puede reciclar. Ello puede redu- cir tanto el reaprovechamiento del papel, del vidrio, del plástico o de las pilas para el mismo u otros fines como el ahorro. Las campañas de los medios de comunicación creadas para concienciar, pueden estar generando un consumo desmedido de algunos productos. En temas de salud, se hacen campañas de prevención con mucho mayor énfasis, pero en temas del medio ambiente, hasta ahora, se han hecho más campañas de reparación (reciclado, reforestación, limpieza, etc.) que de prevención. Con el aumento de la sequía o de los incendios forestales, están aumentando las campañas de ahorro y prevención en estos aspectos concretos, pero no en otros.

En los alumnos del grupo 2, en cambio, están muy igualadas las medidas de amortización y prevención, pero comparándolas con el grupo 1, tienen menor número de sujetos que se refieran a estas medidas. Los alumnos del grupo 3 no las distinguen claramente. 
Estas diferencias pueden deberse a las características socioeconómicas de las tres localidades estudiadas.

Los datos obtenidos en las tablas de grados de importancia de la actividad final parecen avalar la hipótesis de que los alumnos receptores de conocimientos de ecología, es decir, de una enseñanza formal de la misma, combinados con actividades de carácter socioeconómico y ético, modifican sus actitudes. Los datos nos muestran que los estudiantes participantes únicamente en un programa de Educación Ambiental sin haber recibido unos contenidos conceptuales de ecología, apenas desvían sus actitudes hacia modelos de Desarrollo Sostenible; el grupo que recibe conocimientos someros cambia moderadamente sus actitudes, y el grupo que recibe una enseñanza formal de la disciplina ecológica modifica claramente sus actitudes hacia un modelo de desarrollo sostenible. Hemos de decir que este modelo se muestra como la opción actitudinal más correcta al contemplar una comunión entre el progreso y el medio ambiente.

Aunque estos resultados, como ya hemos comentado, parecen confirmar las hipótesis, no obstante, pensamos que a la hora de diseñar un programa de Educación Ambiental, hemos de tener más en cuenta las características socio-económicas del grupo sobre el que se va a aplicar, porque "las consciencias son ecologizadas, sociologizadas, historizadas de formas diversas" (Morín, 2003:65). El grupo que más ha modificado las actitudes hacia modelos de desarrollo sostenible tenía unas características eminentemente rurales, y la actividad final que consistía en la instalación de una fábrica papelera en un pueblo de la ribera del Ebro, quedaba más alejado de su realidad cotidiana que en los otros dos casos, los cuales sí han observado la instalación de industrias diversas en los alrededores. Por otra parte, el hecho de que los estudiantes tuvieran también distinta formación enriquece la investigación, puesto que incluso desde el principio, los estudiantes del grupo 1 quienes tampoco habían recibido enseñanza formal de la ecología hasta ese momento, muestran más actitudes hacia el desarrollo sostenible que los otros grupos. Ello nos permite pensar que podría ser debido a que habían recibido más conocimientos conceptuales de otras disciplinas relacionadas directa o indirectamente con la Ecología como pueden ser la Geología, Biología, Física, Química y Filosofía, lo cual sería un dato a tener en cuenta a la hora de valorar la importancia de la interdisciplinariedad. Pensamos, por tanto, que es importante fomentar la interdisciplinariedad de la Educación Ambiental, así como su tratamiento de forma transversal en un trabajo por competencias. Los trabajos escolares de percepción medioambiental potencian el aprendizaje significativo intrínseco (Morales et al. 2011a) mientras que las experiencias vertebradas a través de instrumentos como la agenda 21 escolar favorecen la concreción de unas pautas de comportamiento medioambientalmente sostenibles ( Ibídem, 2011b), pautas de comportamiento deseables en otros contextos sociales,"porque el legado natural propio es un aval imprescin- 
dible para garantizar la sostenibilidad en el futuro" (Morales, 2002: 476)

\section{Bibliografía}

ALMENAR, R.; BONO, E. y GARCÍA, E (2000) La sostenibilidad del desarrollo: el caso valenciano. Valencia: Universitat de València \& Fundació Bancaixa. València, $575 \mathrm{p}$.

BLISS, J., OGBORN, J., GRIZE, F. (1979). The analysis of Qualitative Data. European Journal in Science Education, Vol.1, nº , pág. 427-440. http://dx.doi.org/10.1080/014052879 0010406

REAL DECRETO 1513/2006, de 7 de diciembre por el que se establecen las enseñanzas mínimas de la Educación primaria (BOE, n ${ }^{\circ} 293$, 08/12/2006)

BOLÍVAR, A. (2010). Competencias básicas y currículo. Síntesis. Madrid, $287 \mathrm{p}$.

CAURÍN C., GIL M.J. (1995). Estudio de las actitudes ambientales en estudiantes de Enseñanza Secundaria. Tesis de tercer ciclo. Universidad de Valencia.

CAURÍN, C. (1999). Análisis, evaluación y modificación de actitudes en Educación Ambiental. Tesis Doctoral, Facultad de Biológicas, Departamento de Didáctica de las Ciencias Experimentales. Universidad de Valencia. Publicaciones. 474 pág.

CAURÍN, C (2001). Los temas transversales en la Cultura Académica. Premio transversales 2000 de la Con- selleria d'Educació. Conselleria de Cultura y Educación de la Generalitat Valenciana.

CAURÍN, C. (2007). Los problemas ambientales y sus repercusiones politicas, económicas y sociales. salud ambiental y calidad de vida. Biología y geología. Ed. CEP. Vol. 3. Tema 51. Madrid

CAURÍN,C., SIGLER, P.(1995) Programa de educación Ambiental para cursos de ESO, Bachilleratos LOGSE y Módulos de Técnicos en Medio Ambiente. Premio Educación y Sociedad del Ministerio de Educación y Ciencia (MEC)

GAVIDIA, V., AGUILAR, R. y CARRATALÁ, A. (2011). ¿Desaparecen las transversales con la aparición de las competencias? Didáctica de las Ciencias Experimentales y Sociales, pág. 131-148. n 25.

HERNÁNDEZ, J. Y CAURÍN, C. (2001). Evaluación de conceptos procedimientos y actitudes ante las energías alternativas en un Centro de Educación Medioambiental. Tesis de tercer ciclo. Universidad de Valencia.

HERNÁNDEZ, J. CAURÍN, C., LLOPIS J. A. (2005). Educación de Actitudes y valores en un contexto no formal. La Problemática de la Energía. Universidad de Valencia.

MARTÍNEZ- PENELLA, M. J., CAURÍN C. y SANJOSÉ V.. (2010). Análisis del concepto de Biodiversidad en los libros de texto de $2^{\circ}$ ciclo de primaria. Trabajo de fin de Máster de Investigación en Didácticas Específicas. Universitat de València 
MORALES, A.J. (2003): Biogeografía y aprovechamiento de la flora autóctona valenciana. Tesis doctorals. Universitat de València, $590 \mathrm{p}$.

MORALES, A. J.; CAURÍN, C..; GARCÍA, F..; ABELLÁN, J. M.; MARCO, $\mathrm{M}^{\mathrm{a}}$. D. y MARCO, N. (febrero, 2011). Claves para la elaboración de un catálogo de percepción florística. VII Congreso Internacional sobre Educación, Cultura y Desarrollo.(CdRom) Universidad de Málaga, Málaga .<http://www.eumed.net/eve/pedirinformes.php $>$

MORALES, A. J.; CAURÍN, C..; GARCÍA, F. y ABELLÁN, J. M. (diciembre, 2011b). La agenda 21 escolar: participación y compromiso del ámbito escolar con el entorno. VIII Congreso Internacional sobre Desarrollo Local en un mundo global.(en línea) Málaga: Universidad de Málaga, febrero de 2011 <http://www.eumed.net/eve/ pedirinformes.php $>$

MORIN, E. (2003). El método. La humanidad de la humanidad. La identidad humana. Anaya. Madrid, 342 p.
ROKEACH, M. (1973): The Nature of Human Values, MacMillan Pub. Co., lnc., N.Y.

SANMARTÍ, N. (1989). Dificultats en la comprensió de la diferenciació entre els conceptes de mescla i compost (Tesi doctoral). Barcelona: Universidad Autónoma de Barcelona.

SOUTO, X.M. (2010). ¿Que escuelas de Geografía para educar en ciudadanía? Didáctica de las Ciencias Experimentales y Sociales, $n^{\circ} 24$. Universitat de València. València, pp 25-44. SUPERKA, D.P. et al (1976): Values Education Sourcebook. Social Science Education Consortium, ERIC Clearinghouse for Social Studies/Social Science Education, Columbus, Ohio.

TURNER, R. AND LEWIS, M. K. Collective Behavior 2d ed. Englewood Cliffs, NJ: Prentice Hall, 1972; 3d ed., 1987; 4th ed., 1993.

WINOGROD (1972). Understanding Natural Language Academic Press, New York. 
